\title{
Evaluation of the "CLARI-RES ASSAY" by real-time for the detection of clarithromycin -resistant Helicobacter pylori in the upper gastrointestinal biopsies
}

\author{
Giorgio Mucignat', Anna Maria Baragiotta², Giorgio Benedetti², Anna Lagatta', Marina Crovatto' \\ I SSD Citogenetica e Biologia Molecolare, Azienda Ospedaliera Santa Maria degli Angeli. Pordenone \\ 2 SSD Gastroenterologia, Azienda Ospedaliera Santa Maria degli Angeli, Pordenone
}

Key words: H. pylori, Claritromicina, Mutazione

Valutazione del kit "CLARI-RES ASSAY" in real-time, per la ricerca di Helicobacter pylori claritromicina resistente su biopsie gastriche

\section{SUMMARY}

Introduction. Clarithromycin is recognized as the main drug of first-line therapy for eradication of Helicobacter pylori (Hp) (Maastricht III Consensus 2006) and in vitro evaluation of its effectiveness is considered crucial. it was noted that some point mutations in domain $V$ of $23 \mathrm{~S}$ rRNA Hp are associated with resistance to macrolides and then is given the possibility of a molecular diagnostic capable, inter alia, to overcome some of the problems associated with classical microbiological techniques. They have been recently commercialized kit employing molecular methods able to identify some of these mutations, which are considered most often. Having assessed by sequencing, the relative frequency of mutations detected in local office, we wanted to compare the potential benefits of molecular diagnostics in comparison to cytological testing in normal use, and assess the frequency of strains with mutations linked to resistance to clarithromycin in population Local.

Methods. 59 patients presenting disorders of the upper gastrointestinal tract were subjected to gastroscopy, biopsies were taken on which it runs parallel with the direct cytology and molecular method. Cytology was performed according to traditional method. The extraction of nucleic acids was performed with QIAamp DNA Mini Kit (Qiagen). The kit "H. pylori ClariRes assay" (Ingenetix GmbH.Vienna) was used for the detection of mutations A2 142C,A2 142/3G by RealTime on LightCycler 2.0 instrument (Roche).

Results. $91.5 \%$ of results are concordant with both methods (54/59). $4 \mathrm{I}$ are positive for the presence of $\mathrm{HP}$ and $\mathrm{I} 8$ are negative. No sample test are positive direct and negative for nucleic acids, vice versa in 5 samples are found DNA of $\mathrm{Hp}$ while the direct examination was negative. Of the 4 I patients positive for Molecular good 19 (46.3\%) appear to be carriers of Hp with mutations linked to resistance Clarithromycin. Conclusions. The molecular method is used and proved sensitive and reliable in clinical practice and to solve technical problems in the crop and in the test of sensitivity on the one hand and the difficulties of interpretation of cytology on the other. The response times are noticeably shorter. Highlighted the frequency of strains resistant to clarithromycin makes it even more this kind to establish a proper antibiotic therapy.

La Claritromicina è riconosciuta essere la colonna portante della terapia di prima scelta per l'eradicazione di Helicobacter pylori (Hp) (Maastricht III consensus 2006) e conseguentemente la valutazione in vitro della sua efficacia è considerata fondamentale per instaurare una corretta terapia. È stato osservato che alcune mutazioni puntiformi nel domain $\mathrm{V}$ del 23S rRNA dell'Hp sono associate a resistenza ai macrolidi. Sono ben 19 le diverse mutazioni puntiformi associate alla resistenza di $\mathrm{Hp}$ ai macrolidi sin qui pubblicate $(\mathrm{A} 2143 \mathrm{G}, \mathrm{A} 2144 \mathrm{G}, \mathrm{A} 2142 \mathrm{G}, \mathrm{A} 2142 \mathrm{C}, \mathrm{T} 2182 \mathrm{C}, \mathrm{A} 2143 \mathrm{C}$, $\underline{\mathrm{T} 2183 \mathrm{C}}, \underline{\mathrm{T} 2717 \mathrm{C}}, \underline{\mathrm{A} 2144 \mathrm{~T}}, \underline{\mathrm{C} 2195 \mathrm{~T}}, \underline{\mathrm{T} 2221 \mathrm{C}}, \mathrm{C} 2147 \mathrm{G}$, C2245T, G1939A, G2224A, T1942C, T2245C, T2289C, C2611A), di queste, le prime 11 (sottolineate) sono state riportate in lavori di autori italiani.

Recentemente sono stati commercializzati dei kit utilizzanti tecniche molecolari in grado di identificare alcune di queste mutazioni, ritenute più frequenti.

Abbiamo quindi valutato il tipo di mutazioni presenti in sede locale per mezzo della tecnica di sequenziamento, per conoscere quanta parte di queste mutazioni fossero evidenziabili con il kit commerciale "ClariRes Assay" e, contemporaneamente, verificare la frequenza di ceppi con mutazioni legate a resistenza a Claritromicina nella popolazione afferente al servizio di Gastroenterologia di Pordenone, per definire la dimensione locale di dette resistenze. È stato inoltre possibile il confronto "sul campo" dei vantaggi/svantaggi di una tecnica molecolare versus il test citologico, attualmente unica diagnostica di uso in loco per la ricerca di Hp su biopsie. Sono stati sin qui arruolati c/o SSD Gastroenterologia dell'Azienda ospedaliera di Pordenone 59 pazienti che presentavano disturbi del tratto gastroenterico superiore, così suddivisi: 29 femmine (49\%) e 30 maschi (51\%); 10 africani
$(17 \%)$ e 49 caucasici (83\%). Età media: $50 \pm 16$.

In corso di EGDS sono stati eseguiti prelievi bioptici di mucosa gastrica per ricerca diretta di spirilli e analisi molecolare per la ricerca di Hp e l'eventuale mutazione .

È stato utilizzato il kit "H. pylori ClariRes Assay" (Ingenetix $\mathrm{GmbH}$. Vienna) per la ricerca delle mutazioni A2142C, A2142/3G mediante RealTime PCR, su strumento LightCycler 2.0 (Roche)

Sequenziamento: Su 52 dei 59 pazienti è stato eseguito il sequenziamento della regione $23 \mathrm{~S}$ rRNA dove si concentrano le mutazioni che conferiscono resistenza alla Claritromicina (macrolidi) per ricercare eventuali altre mutazioni non evidenziate dal Kit "ClariRes Assay" in RealTime PCR. Il sequenziamento dei prodotti è stato eseguito con elettroforesi capillare (ABI Prism, Applied Biosistem). Le sequenze sono state analizzate con il GENE SEQUENCING software ABI PRISM e con BLAST.

L'esame citologico è stato eseguito secondo metodica classica.

L'estrazione degli acidi nucleici dalle biopsie è stata eseguita con QIAamp DNA Mini Kit (QiaGen).

In totale 41 su 59 campioni sono risultati positivi per la presenza di Hp. Dei 52 compioni sottoposti a sequenziamento: 17 campioni RealTime PCR negativi sono risultati negativi anche con il Sequenziamento; 20 campioni RealTime PCR $\mathrm{Hp}+\mathrm{S}$ non hanno presentato mutazioni nel Sequenziamento; dei 15 campioni RealTime PCR Hp+R, 13 non hanno presentato altre mutazioni, mentre in 2 non sono state ritrovate mutazioni. Dei 15 campioni RealTime PCR Hp+R, 10 presentavano la mutazione $\mathrm{A} 2143 \mathrm{G}$ e 5 la mutazione A2142G.

$\mathrm{Nel}$ raffronto tra test molecolare vs citologico i risultati risultano concordanti nel $91.5 \%$ dei casi, nessun campione risulta

\section{Corresponding author: Giorgio Mucignat}

A.O. Santa Maria degli Angeli - Servizio Citogenetica e Biologia molecolare Via Montereale 24, 33 I 70 Pordenone - Tel.: 0434399645 - Fax: 0434399685 E-mail: giorgio.mucignat@aopn.fvg.it 
positivo all'esame citologico e negativo alla ricerca di acidi nucleici di $\mathrm{Hp}$.

\section{Spirilli presenti Spirilli assenti}

\begin{tabular}{llcc}
\hline Molecolare & & \\
\hline $\mathrm{Hp}$ & Hp presente & 36 & $4+\mathrm{I}^{*}$ \\
\hline & Hp assente & 0 & 18 \\
\hline
\end{tabular}

* caso dubbio

4 I campioni sono risultati positivi (69.4\%). Di questi 22 sono risultati Wild-type mentre 19 (46.3\%) presentano mutazioni legate a resi-

La distribuzione delle caratteristiche tra $\mathrm{i}$ due gruppi $\mathrm{HpR} / \mathrm{HpS}$ di pazienti è la seguente: stenza ai macrolidi.

rRNA ci ha assicurati sulla esclusiva presenza di queste sole mutazioni nel nostro campione.

In due casi, tuttavia non sono state ritrovate le mutazioni evidenziate con il kit a causa della minore sensibilità del sequenziamento rispetto al metodo molecolare Real Time PCR.

Il metodo molecolare usato si è rivelato sensibile ed affidabile nella pratica clinica ed in grado di risolvere le difficoltà tecniche del metodo microbiologico colturale e dell'esame citologico.

Il metodo molecolare è ovviamente più sensibile e standardizzabile dell'esame citologico ed inoltre aggiunge il dato sulla suscettibilità all'antibiotico cardine della terapia a tutt'oggi maggiormente consigliata.

L'esame citologico rimane comunque il solo in grado di fornire indicazioni sul reale stato di alterazione della mucosa. È da sottolineare come la presenza di mutazioni riconducibili a resistenza alla Claritromicina

\begin{tabular}{llll}
\hline $\begin{array}{l}\text { Pazienti Hp Cla R } \\
\text { (19/4I) }\end{array}$ & \multirow{2}{*}{$46 \%$} & Hp istologia -ve 2/19 & $10.5 \%$ \\
\cline { 3 - 4 } & & Fallimento ter. eradicante $13 / 19$ & $68 \%$ \\
\hline & & & $10.5 \%$ \\
\hline $\begin{array}{lll}\text { Pazienti Hp Cla S } \\
\text { (22/4I) }\end{array}$ & \multirow{2}{*}{$54 \%$} & Hp istologia -ve 3/22 & $14 \%$ \\
\cline { 2 - 3 } & & Fallimento ter. eradicante $4 / 22$ & $18 \%$ \\
\hline
\end{tabular}

Le mutazioni trovate sono state quindi solamente due: A2143G e A2142G. Il sequenziamento della regione 23S abbia comportato un fallimento terapeutico del $68 \%$ dei pazienti contro il $18 \%$ di quelli in cui non sono state ritrovate queste mutazioni.

Data l'elevata frequenza di mutazioni rilevate associate a resistenza alla Claritromicina presenti nel $46 \%$ dei soggetti valutati (di cui $30 \%$ c.a. naïve), riteniamo importante, in accordo con la recente letteratura (Mégraud F. and Lehours 2007), la valutazione della resistenza prima di instaurare la terapia per l'eradicazione di Helicobacter pylori.

\section{BIBLIOGRAFIA}

1. Bruce MG, Bruden DL, McMahon BJ, et al. Alaska sentinel surveillance for antimicrobial resistance in Helicobacter pylori isolates from Alaska native Persons, 1999-2003. Helicobacter 2006; 11: 581-8.

2. De Francesco V, Margiotta M, Zullo A, et al.. Clarithromycin-resistant genotypes and eradication of H. pylori. Ann Intern Med 2006; 144: 94-100.

3. Fontana C, Favaro M, Minelli S, et al. New site of modification of $23 \mathrm{~S}$ rRNA associated with clarithromycin resistance of Helicobacter pylori clinical isolates. Antimicrob Agents Chemother 2002; 46: 3765-9.

4. Hao Q, Li Y, Zhang ZJ, et al. New mutations points in $23 \mathrm{~S}$ rRNAgene associated with Helicobacter pylori resistance to clarithromycin in northeast China. World J Gastroenterol 2004; 10: 1075-7.

5. Khan R, Nahar S, Sultana J, et al. T2182C mutation in $23 \mathrm{~S}$ rRNA is associated with clarithromycin resistance in Helicobacter pylori isolates obtained in Bangladesh. Antimicrob Agents Chemother 2004; 48:3567-9.

6. Kim J M. Antibiotic resistance of H. pylori isolated from Korean patients. Korean J. Gastroenter. 2006; 47:337-49.

7. Kim J, Mogg J, Kim JS, Kim N, et al. Gene Mutations of 23S rRNA Associated with Clarithromycin Resistance in Helicobacter pylori Strains Isolated from Korean Patients J. Microbiol. Biotechnol. 2008; 18(9), 1584-89

8. Liu Z, Shen J, Zhang L, et al. Prevalence of A2143G mutation of H. pylori23S rRNA in Chinese subjects with and without clarithromycin use history. BMC Microbiology 2008; 8:81
9. Liu ZQ,Zheng PY,Yang PC. Efflux pump gene hefA of H. pylori plays an important role in multidrug resistance. World J Gastroenterol 2008; 14 : 5217-22

10. Mégraud F, Lehours H. pylori Detection and Antimicrobial Susceptibility Testing. Clin Microb Rew 2007; 280-322.

11. Mégraud F. H pylori antibiotic resistance: prevalence, importance, and advances in testing. Gut 2004; 53: 1374-84.

12. National Committee for Clinical Laboratory Standards. Performance standards for antimicrobial susceptibility testing. Twelfth Informational Supplement: M100-S12. NCCLS, Wayne, PA, USA, 2002.

13. Oleastro M, Menard A, Santos A, et al. Real-time PCR assay for rapid and accurate detection of point mutations conferring resistance to clarithromycin in Helicobacter pylori. J Clin Microbiol 2003; 41:397-402.

14. Ribeiro ML, Vitiello L, Miranda MCB, et al. Mutations in the 23S rRNA gene are associated with clarithromycin resistance in Helicobacter pylori isolates in Brazil. Ann Clin Microbiol Antimicrob 2003; 2: 1-4.

15. Sanger F, Nicklen S, Coulson A. DNA sequencing with chain-terminating inhibitors. Proc Nat Acad Sci US 1997; 74: 5463-7.

16. Taylor DE, Ge Z, Purych D, et al. Cloning and sequence analysis of two copies of a 23S rRNA gene from Helicobacter pylori and association of clarithromycin resistance with $23 \mathrm{~S}$ rRNA mutations. Antimicrob Agents Chemother 1997; 41: 2621-8.

17. Toracchio S, Aceto GM, Mariani-Costantini R, et al. Identification of a novel mutation affecting domain $\mathrm{V}$ of the $23 \mathrm{~S}$ rRNA gene in Helicobacter pylori. Helicobacter 2004; 9: 396-9. 